\title{
Silencing Tspan1 inhibits migration and invasion, and induces the apoptosis of human pancreatic cancer cells
}

\author{
JIAXUN TIAN ${ }^{1}$, RUI ZHANG ${ }^{2}$, HAIYAN PIAO ${ }^{3}$, XIAOXI LI $^{3}$, WEIWEI SHENG ${ }^{4}$, JIANPING ZHOU ${ }^{4}$, MING DONG $^{4}$, \\ XIAOBO ZHANG ${ }^{5}$, XIAOFEI YAN ${ }^{2}$, WEN SHANG ${ }^{2}$, JIANFENG ZHAO ${ }^{2}$, LAN XU $^{1}$, FANG LIU ${ }^{2}$ and GANG SHI ${ }^{2}$ \\ Departments of ${ }^{1}$ Laboratory, ${ }^{2}$ Colorectal Surgery and ${ }^{3}$ Medical Oncology, Cancer Hospital of China Medical University, \\ Liaoning Cancer Hospital and Institute, Shenyang, Liaoning 110042; ${ }^{4}$ Gastrointestinal Surgery Department, \\ The First Affiliated Hospital of China Medical University; ${ }^{5}$ General Surgery Department, \\ Shengjing Hospital of China Medical University, Shenyang, Liaoning 110001, P.R. China
}

Received May 23, 2017; Accepted March 5, 2018

DOI: $10.3892 / \mathrm{mmr} .2018 .9331$

\begin{abstract}
Pancreatic cancer (PCC) is one of the most dangerous types of tumor as it is very difficult to treat and its 5 -year survival rate is $<6 \%$. To date, there have been no effective therapeutic strategies to treat PCC, thus, novel effective therapeutic methods are required. Tetraspanin 1 (Tspan1) is a novel member of the tetraspanins superfamily and is highly expressed in a variety of types of cancer, including gastric, hepatocellular and colonic carcinomas. However, the detailed functional role of Tspan1 in pancreatic cancer cells is still unclear and further investigation is required to uncover its therapeutic potential for the treatment of different tumor types. The purpose of the present study was to investigate the expression of Tspan1 in human PCC tissues and cells, and explore the effect of Tspan1 silencing on invasion, migration, cell survival and apoptosis in human PCC to clarify its function. Expression levels of Tspan1 were analyzed in human pancreatic cancer tissues and the cell lines Capan-2 and SW1990 using immunohistochemistry staining, reverse transcription-quantitative polymerase chain reaction and western blotting. The effects of downregulation of Tspan1 expression on cell survival, apoptosis, invasion and migration were investigated viaTspan1-small interfering (si)RNA transfection into human PCC cell lines. The results indicated that Tspan1 expression was increased in human PCC tissues compared with the adjacent normal pancreatic tissues. Tspan1 was highly expressed in the human PCC cell lines Capan-2 and SW1990 when compared with the normal pancreatic cell line HPC-Y5. In addition, transfection with siRNA-targeting
\end{abstract}

Correspondence to: Dr Gang Shi, Department of Colorectal Surgery, Cancer Hospital of China Medical University, Liaoning Cancer Hospital and Institute, 44 Xiaoheyan Road, Shenyang, Liaoning 110042, P.R. China

E-mail: shigang@cancerhosp-ln-cmu.com

Key words: tetraspanin 1, migration, invasion, human pancreatic cancer, cells apoptosis, proliferation
Tspan1 significantly reduced cell migration and invasion, and increased the cell apoptosis of Capan-2 and SW1990. The present findings highlighted the important role of Tspan1 in human PCC cell migration, invasion and apoptosis. Thus, Tspan1 RNA interference may serve as a potential therapeutic strategy to treat human PCC.

\section{Introduction}

Pancreatic cancer (PCC) is currently one of the leading causes of cancer-associated mortality worldwide, with pancreatic ductal adenocarcinoma (PDAC) accounting for $80-90 \%$ of pancreatic tumor cases $(1,2)$. The 5-year survival rate of PDAC patients is $<6 \%$ and the average survival time of PDAC patients is $<6$ months (2). The majority of patients are diagnosed in the advanced stages of cancer, and succumb due to cancer invasion and migration $(3,4)$. In addition, the majority of patients with PDAC do not have long-term survival rates with the current therapeutic methods, which include surgical resection, radiation and chemotherapy (5-8). Therefore, the development of novel and effective therapeutic strategies is urgently required for the effective treatment of PDAC.

The tetraspanins heterogeneous superfamily (TM4SF) is composed of tetraspanin-enriched microdomains (TEMs). TM4SF has four transmembrane regions that interact with a number of cell surface signaling molecules, such as integrins $(9,10)$. It has been reported that TEMs are involved in many fundamental biological processes, including cell proliferation, adhesion, proliferation, migration and signaling transduction $(11,12)$. Tetraspanin 1 (Tspan1) is a novel member of the tetraspanins family, which is reported to be located at chromosome 1 p34.1 and encodes a protein with 241 amino acids (13). Previous studies have demonstrated that Tspan1 is overexpressed in various tumor cells including cervical, lung, gastric, squamous cell and colon carcinomas, and liver and breast cancers (14-18); however, few studies have interpreted its role in PDAC. Xu et al (19) identified a differentially expressed Tspan1 gene in human prostate tissues and prostate cancer using cDNA database subtraction and microarray analysis. In addition, previous studies revealed that Tspan1 regulated human cancer progression in non-small cell lung and colon 
carcinomas, and skin squamous and cervical cancers (20-24). However, the detailed functional role of Tspan1 in human PCC is still unclear.

In the present study, the expression of Tspan1 in human pancreatic cancer tissues, adjacent normal pancreatic tissues and human PDAC cell lines were detected using immunohistochemistry (IHC), reverse transcription-quantitative polymerase chain reaction (RT-qPCR) and western blotting. Subsequently, following the transfection of Tspan1 small interfering (si)RNAs into human PCC cells, the expression of Tspan1 was analyzed by western blotting and RT-qPCR. Cell apoptosis and cell survival were detected by flow cytometry and an MTT assay. In addition, the effect of Tspan1 silencing on cell migration and invasion were explored using a Transwell assay.

\section{Materials and methods}

Tissues, cell lines and cell culture. The human PDAC cell lines Capan-2 and SW1990, and normal human pancreatic cell line HPC-Y5 were purchased from Shanghai Institute of Biochemistry and Cell Biology, Chinese Academy of Sciences (Shanghai, China). The cells were grown in Dulbecco's modified Eagle's medium (DMEM; Invitrogen; Thermo Fisher Scientific, Inc., Waltham, MA, USA) supplemented with $10 \%$ fetal calf serum (FCS; Invitrogen; Thermo Fisher Scientific, Inc.). The cells were cultured in DMEM at $37^{\circ} \mathrm{C}$ with $5 \% \mathrm{CO}_{2}$ and passaged when $70-80 \%$ confluent using $0.25 \%(\mathrm{w} / \mathrm{v})$ trypsin solution in $0.04 \%(\mathrm{w} / \mathrm{v})$ EDTA. A total of 20 pairs of human tumor and adjacent normal pancreatic tissues were obtained from the Department of Gastrointestinal Surgery of the First Affiliated Hospital of China Medical University (Shenyang, China) once official written informed consent was received from each patient. A total of 95 patients were recruited between June 2015 and June 2016 (52 males and 43 females; aged 39-81 years old). Included patients had confirmed PCC diagnoses and had not previously undergone radiation or chemotherapy. During routine surgery performed in the Gastrointestinal Surgery Department of Cancer Hospital of China Medical University (Shenyang, China) and the Gastrointestinal Surgery Department of First Affiliated Hospital of China Medical University (Shenyang, China), cancer tissues and adjacent normal pancreatic tissues were collected. The present study was approved by the Ethical Committee of China Medical University.

IHC. Anti-TSPAN1 antibody (1:200; cat no. NBP2-33867; Novus Biologicals, LLC, Littleton, CO, USA) was used for detecting TSPAN1expression with IHC staining. Tissue sections embedded in paraffin $(4 \mu \mathrm{m})$ were sequentially deparaffinized and rehydrated, then antigens were retrieved at $95^{\circ} \mathrm{C}$ using 10 mM citrate buffer (pH 6.0; Merck KGaA, Darmstadt, Germany). Freshly prepared $3 \% \mathrm{H}_{2} \mathrm{O}_{2}$ was used to quench the endogenous peroxidase activity. Normal serum (10\%; Invitrogen; Thermo Fisher Scientific, Inc.) was used to block non-specific staining at room temperature for $1 \mathrm{~h}$. Subsequently, sections were incubated with anti-TSPAN1 primary antibodies (1:500; cat.no. NBP2-33867; Novus Biologicals, LLC) at $37^{\circ} \mathrm{C}$ for $2 \mathrm{~h}$, which was followed by incubation with goat anti-rabbit IgG biotinylated antibodies (1:1,000; cat.no. BAF008; R\&D
Systems, Inc., Minneapolis, MN, USA) at room temperature for $30 \mathrm{~min}$. Following this, sections were washed with PBS and then incubated with horseradish peroxidase-conjugated streptavidin (1:2,000; cat.no. N100; Thermo Fisher Scientific, Inc.) for $5 \mathrm{~min}$ at room temperature. 3,3-Diaminobenzidine substrate (Gene Tech Co., Ltd.) was used to develop the immunostaining for $10 \mathrm{~min}$ at room temperature. Finally, sections were counterstained with hematoxylin (Invitrogen; Thermo Fisher Scientific, Inc.) at room temperature for $3 \mathrm{~min}$. Sections were incubated with PBS as negative control. The cells were examined under an inverted-TS100 microscope (Nikon Corporation, Tokyo, Japan). Furthermore, staining intensity was scored using the following criteria: \pm (negative),+ (weak), ++ (intermediate) and +++(strong) (25).

$R T-q P C R$. Briefly, RNA was extracted from tissues (100 mg) using $1 \mathrm{ml}$ Trizol (Invitrogen Life Technologies), according to the manufacturer's instructions. Following this, tissues were homogenized and subsequently incubated at room temperature for $5 \mathrm{~min}$. Following chloroform (Sigma-Aldrich; Merck $\mathrm{KGaA}$ ) extraction and precipitation with isopropanol (Thermo Fisher Scientific, Inc.), the RNA pellet was washed with $75 \%$ ethanol (Sigma-Aldrich; Merck KGaA), air-dried and subsequently dissolved in nuclease-free water (30 $\mu \mathrm{l}$; Ambion; Thermo Fisher Scientific, Inc.). RNA from cells was obtained using an RNeasy Mini kit (Qiagen GmbH, Hilden, Germany) following the manufacturer's protocol. Reverse transcription was performed as follows: A mixture of $2 \mu \mathrm{g}$ total RNA and $1 \mu \mathrm{l}$ Oligo (dT) was incubated at $70^{\circ} \mathrm{C}$ for $5 \mathrm{~min}$, rapidly chilled on ice and subsequently added to a reaction mixture containing $5 \mu \mathrm{l}$ M-MLV RT 5X Buffer (Promega Corporation, Madison, WI, USA), $0.5 \mu \mathrm{l}$ deoxyribonucleotide triphosphate (25 $\mu \mathrm{M}$; Promega Corporation), $0.7 \mu \mathrm{l}$ RNA inhibitors (Promega Corporation) and $1 \mu \mathrm{l}$ M-MLV reverse transcriptase (200 U/ $\mu 1$; Promega Corporation). The reaction volume was made up to $25 \mu \mathrm{l}$ with RNA-free water and subsequently incubated at $42^{\circ} \mathrm{C}$ for $1 \mathrm{~h}$ and then $70^{\circ} \mathrm{C}$ for $10 \mathrm{~min}$. RT was performed on an Applied Biosystems 7900HT thermal cycler instrument (Applied Biosystems; Thermo Fisher Scientific, Inc.) according to the manufacturer's protocol (26). Briefly, qPCR was carried out in a $20 \mu 1$ reaction volume including 2XMix SYBR green I (10 $\mu \mathrm{l}$; Promega Corporation), primer $(0.25 \mu \mathrm{l}, 10 \mathrm{pmol} / \mathrm{l})$, template DNA $(1 \mu \mathrm{l})$ and sterile water. The thermocycling conditions were as follows: Initial denaturation at $95^{\circ} \mathrm{C}$ for $2 \mathrm{~min}$, followed by 50 cycles of $95^{\circ} \mathrm{C}$ for $15 \mathrm{sec}$ and $60^{\circ} \mathrm{C}$ for $45 \mathrm{sec}$. Fold change of target gene expression levels were calculated using the $2^{-\Delta \Delta \mathrm{Cq}}$ method (27). The following primer sequences were used: Human Tspanl forward, 5'-CGT TGTGGTCTTTGCTCTTG-3' and reverse, 5'-TTCTTGATG GCAGGCACTAC-3'; Human GAPDH forward, 5'-GAA GGTGAAGGTCGGAGTC-3' and reverse, 5'-GAAGATGGT GATGGGATTTC-3'. The relative Tspan1 mRNA expression level was normalized to the endogenous GAPDH mRNA expression.

Western blotting. Radioimmunoprecipitation Assay buffer (Sigma-Aldrich; Merck KGaA) as well as Protease Inhibitor (Sigma-Aldrich; Merck KGaA) were used to lyse cells (28). Following protein collection, a BCA Pierce Assay (Thermo Fisher Scientific, Inc.) was used to quantify the protein 
concentration. Protein $(50 \mu \mathrm{g})$ from each sample was denatured and resolved by 10\% SDS-PAGE (EMD Millipore, Billerica, MA, USA) and electroblotted to a polyvinylidene fluoride (PVDF) membrane (EMD Millipore). PVDF membranes were incubated with $5 \%$ non-fat milk for one hour at room temperature and were then incubated with anti-TSPAN1 antibody (1:300; cat.no. NBP2-33867; Novus Biologicals, LLC, Littleton, CO, USA) or anti- $\beta$-actin antibody (1:1,000; cat.no. NB600-503; Novus Biologicals, LLC) at $4^{\circ} \mathrm{C}$ overnight. Following washing with TBS with 5\% Tween-20, the membrane was incubated with horseradish peroxidase-conjugated secondary antibodies (1:3,000; cat.no. NB710-57836; Novus Biologicals, LLC) for $1.5 \mathrm{~h}$ at room temperature. Finally, signals were developed by enhanced chemiluminescence (Pierce; Thermo Fisher Scientific, Inc.). The optical density of each protein band was quantified by a scanning densitometer and Quantity One software (version 4.4.1; Bio-Rad Laboratories, Inc., Hercules, CA, USA). Each lane of protein band density was normalized against corresponding $\beta$-actin densities.

Knockdown of Tspan1 in SW1990 and Capan-2 cells. Expression of Tspan1 in SW1990 and Capan-2 cells was silenced using specific Tspan1 siRNAs [(Tspan1 siRNA-1 and siRNA-2 (assay IDs were S19659 and S19660, respectively; cat.no. 4392420; Thermo Fisher Scientific, Inc.)]. Scramble siRNA (siNC; cat.no. 4390846; Thermo Fisher Scientific, Inc.) was used to confirm the specificity of Tspan1 siRNAs. Non-transfected cells were used as a negative control. The Tspan1 siRNA-1 group sequences were 5'-GGCUCACGA CCAAAAAGUAtt-3' (sense) and 5'-UACUUUUUGGUC GUGAGCCtt-3' (antisense). The Tspan1 siRNA-2 group sequences were: 5'-GCUUUUGUAUGACAUCCGAtt-3' (sense), 5'-UCGGAUGUCAUACAAAAGCtg-3' (antisense). The scramble siRNA group sequences were 5'-UUCUCC GAACGUGUCACGUdTdT-3' (sense), 5'-ACGUGACACGUU CGGAGAAdTdT-3' (antisense). The siRNAs transfection into Capan-2 and SW1990 cells was performed following the manufacturer's protocol. Briefly, a total of $1 \times 10^{5}$ cells $/ \mathrm{ml}$ of SW1990 and Capan-2 cells were re-suspended in DMEM medium. Transfection complexes were prepared by mixing RNAiMAX Lipofectamine transfection agent (Ambion; Thermo Fisher Scientific, Inc.) and Tspan1 siRNA (20 nM) or scramble siRNA (20 nM) in DMEM medium. SW1990 and Capan-2 cells, and the transfection complexes were mixed and incubated for $24 \mathrm{~h}$ at $37^{\circ} \mathrm{C}$ in 6 -well $\left(2 \times 10^{5}\right.$ cells/well) plates (NalgeNunc International, Penfield, NY, USA). Cells were collected at $48 \mathrm{~h}$ post-transfection for RNA analysis and $72 \mathrm{~h}$ for protein analysis. The knockdown of Tspan1 was confirmed by RT-qPCR and western blot analysis.

In vitro cell migration and invasion assay. The cell migration was detected using the BD Falcon HTS multi-well insert system (pore size, $8.0 \mu \mathrm{m}$ ) (29). A total of $1.5 \times 10^{5} \mathrm{SW} 1990$ and Capan-2 cells, or transfected SW1990 and Capan-2 cells in serum-free DMEM were plated in the upper chamber of the insert; in the bottom wells $500 \mu \mathrm{l}$ DMEM containing $10 \%$ FCS was plated. Following incubation for $6 \mathrm{~h}$ at $37^{\circ} \mathrm{C}$, cotton swabs were used to carefully scrape off the non-migrated cells (the cells remaining in the insert). The migrated cells (the cells at the bottom of the insert) were fixed with $4 \%$ formaldehyde for $20 \mathrm{~min}$ at room temperature, and then stained with $0.1 \%$ crystal violet for $30 \mathrm{~min}$ at room temperature. Milli-Q water was used to wash away the excess stain and the filters were dried overnight. Migrated cells were manually counted in five random fields and images were obtained using light microscopy and camera (Nikon Digital Camera Dxm 1200F; Nikon Corporation).

The cell invasion assay was performed using the BioCoat Matrigel invasion chambers (BD Biosciences, Franklin Lakes, NJ, USA) (30). Reduced serum Matrigel (50 $\mu \mathrm{g}$; BD Biosciences) was added to coat Transwell inserts. Following transfection, a total of $1 \times 10^{6}$ SW1990 and Capan- 2 cells in serum-free DMEM were plated in the upper chamber. DMEM with $10 \%$ FCS was plated in the lower chamber as the chemoattractant. Following $48 \mathrm{~h}$ of incubation at $37^{\circ} \mathrm{C}$, the membrane containing the invaded cells was taken off gently and mounted on a glass slide, then the slide was fixed with $4 \%$ formaldehyde for $20 \mathrm{~min}$ at room temperature and stained with $0.1 \%$ crystal violet at room temperature for $30 \mathrm{~min}$. Invaded cells were counted in five random fields and images obtained using light microscopy and camera (Nikon Digital Camera Dxm 1200F; Nikon Corporation).

Flow cytometric analysis of apoptosis. The Alexa Fluor ${ }^{\circledR}$ Annexin V/Dead Cell Apoptosis kit (Invitrogen; Thermo Fisher Scientific, Inc.) was used to detect the number of apoptotic cells. A total of $8 \times 10^{5}$ cells were seeded into 6-well plates. Following siRNA transfection, cells were collected, washed with cold PBS and then re-suspended in $100 \mu l$ binding buffer (BB; Invitrogen; Thermo Fisher Scientific, Inc.). Following this, Annexin V (5 $\mu \mathrm{l})$ and propidium iodide (PI;10 $\mu \mathrm{l})$ were added. The cells were incubated for $30 \mathrm{~min}$ in darkness at room temperature. Finally, cells were washed twice in BB. A FACS Calibur flow cytometer (BD Biosciences) in conjunction with CellQuest software (version 5.1; BD Biosciences) were used to determine the fluorescence intensity of labeled cells and to calculate the percentage of apoptotic cells.

Cell survival (MTT) assay. To investigate the effect of Tspan1 downregulation on the proliferation and viability of SW1990 and Capan-2 cells, MTT assays were performed at $0,24,48,72,96$ and $120 \mathrm{~h}$ time intervals following transfection. A total of $3 \times 10^{4}$ cells were seeded into 96 -well plates a day prior to transfection. Cells were cultured in a $5 \% \mathrm{CO}_{2}$ incubator at $37^{\circ} \mathrm{C}$ for $4 \mathrm{~h}$ with $150 \mu 1$ DMEM containing $20 \mu 1$ MTT (5 mg/ml; Sigma-Aldrich; Merck KGaA) per well. Following incubation, the medium was then gently aspirated and $150 \mu \mathrm{l}$ of dimethyl sulfoxide (Sigma-Aldrich; Merck KGaA) was subsequently added to each well to solubilize the formazan crystals. The absorbance of formazan was determined at a wavelength of $570 \mathrm{~nm}$ and a reference wavelength of $630 \mathrm{~nm}$ using a Bio-Rad Microplate Reader 550 (Bio-Rad Laboratories Inc.). Cell growth curves were subsequently plotted using the mean \pm standard deviation data.

Statistical analysis. The results were expressed as the mean \pm standard deviation of at least three independent experiments performed in triplicate. The difference between two groups was analyzed using the two-tailed Student's t-test and 
A

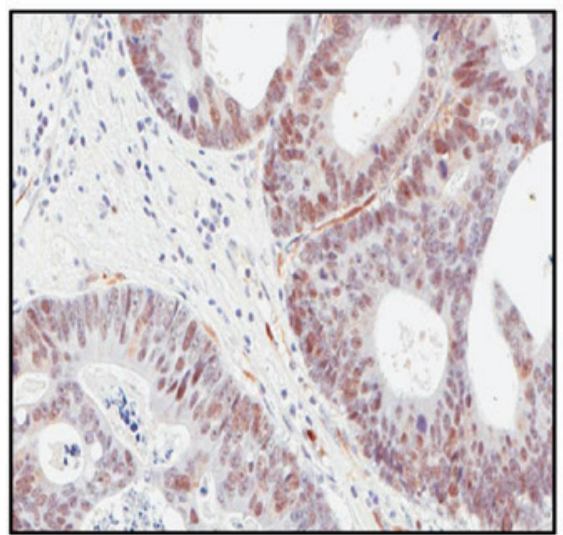

B

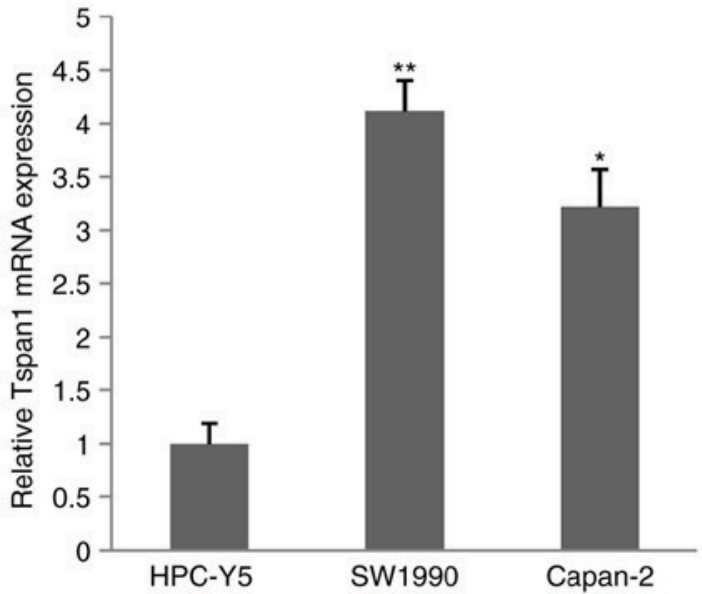

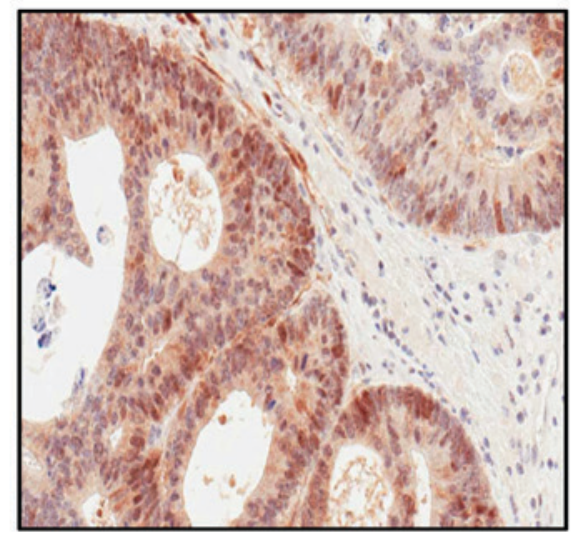
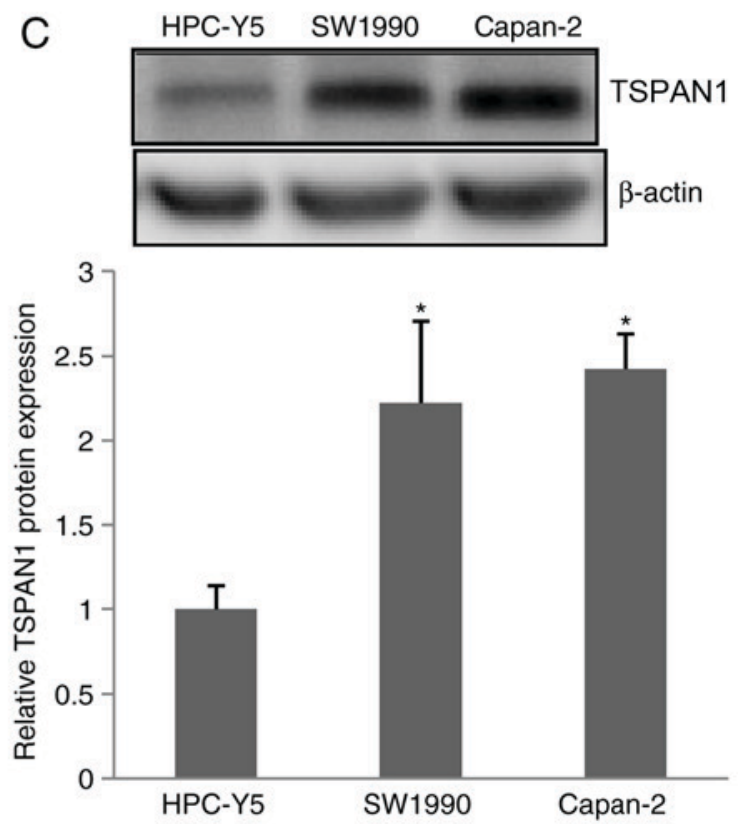

Figure 1. Expression of TSPAN1 in human pancreatic tumor tissues and cells. (A) Representative images of pancreatic cancer tissues (right) and adjacent non-cancer tissues (left), as detected by immunohistochemistry. Magnification, x200. Quantification of Tspan1 (B) mRNA and (C) protein expression levels, in the human pancreatic cancer cell lines SW1990 and Capan-2, and normal pancreatic cell line HPC-Y5, as examined by reverse transcription-quantitative polymerase chain reaction and western blot analysis, respectively. Data are expressed as the mean \pm standard deviation. ${ }^{*} \mathrm{P}<0.05$ and ${ }^{* *} \mathrm{P}<0.01$ vs. $\mathrm{HPC}-\mathrm{Y} 5$ (n=3). TSPAN1, Tetraspanin 1 .

SPSS 17.0 software (SPSS, Inc., Chicago, IL, USA). One-way analysis of variance followed by the Tukey's post hoc test were used to assess significant differences among groups by SPSS 17.0 software (SPSS, Inc.). $\mathrm{P}<0.05$ was considered to indicate a statistically significant difference.

\section{Results}

Expression of Tspanl in human PCC tissues and cells. IHC was used to detect the expression status of TSPAN1 in human carcinoma and adjacent normal pancreatic tissues. The results indicated that there were greater levels of TSPAN1 protein in PCC tissues (Fig. 1A, right) than there were in the adjacent normal pancreatic tissues (Fig. 1A, left). Positive TSPAN1 immunostaining was mainly localized in the cytoplasm of carcinoma tissue cells. The statistical analysis results of TSPAN1 expression in human pancreatic tumor tissues and adjacent normal pancreas tissues are presented in Fig. 2. The mRNA expression levels of Tspan1 were measured in the PDAC cell lines Capan-2 and SW1990, and the normal pancreatic cell line HPC-Y5 using RT-qPCR. The results demonstrated that the SW1990 and Capan-2 cells expressed higher levels of Tspan 1 mRNA when compared with the normal pancreatic cell line HPC-Y5 (Fig. 1B). TSPAN1 protein expression was also detected using western blotting. The results revealed that the SW1990 and Capan-2 cells expressed higher levels of TSPAN1 protein compared with the normal pancreatic cell line HPC-Y5 (Fig. 1C).

Knocking down Tspanl expression by siRNA transfection. The human PDAC cell lines SW1990 and Capan-2 were transfected with Tspan1-siRNAor siNC, which was used to confirm the specificity of Tspan1 siRNAs. Tspan1 mRNA and protein expression levels were determined using RT-qPCR and western blotting, respectively, to confirm that its expression was silenced. The results revealed that there were no differences in Tspan1 expression levels between siNC cells and untransfected control cells (Fig. 3). RT-qPCR results 
A Table 1. Relative expression of TSPAN1

\begin{tabular}{|c|c|c|c|c|c|c|}
\hline Group & Number & \multicolumn{4}{|c|}{ TSPAN1 expression intensity } & P \\
\hline $\begin{array}{c}\text { Pancreatic tumor } \\
\text { tissues }\end{array}$ & 20 & 2 & 3 & 8 & 7 & $<0.01$ \\
\hline $\begin{array}{c}\text { Adjacent normal } \\
\text { pancreas tissues }\end{array}$ & 20 & 15 & 5 & 0 & 0 & \\
\hline
\end{tabular}

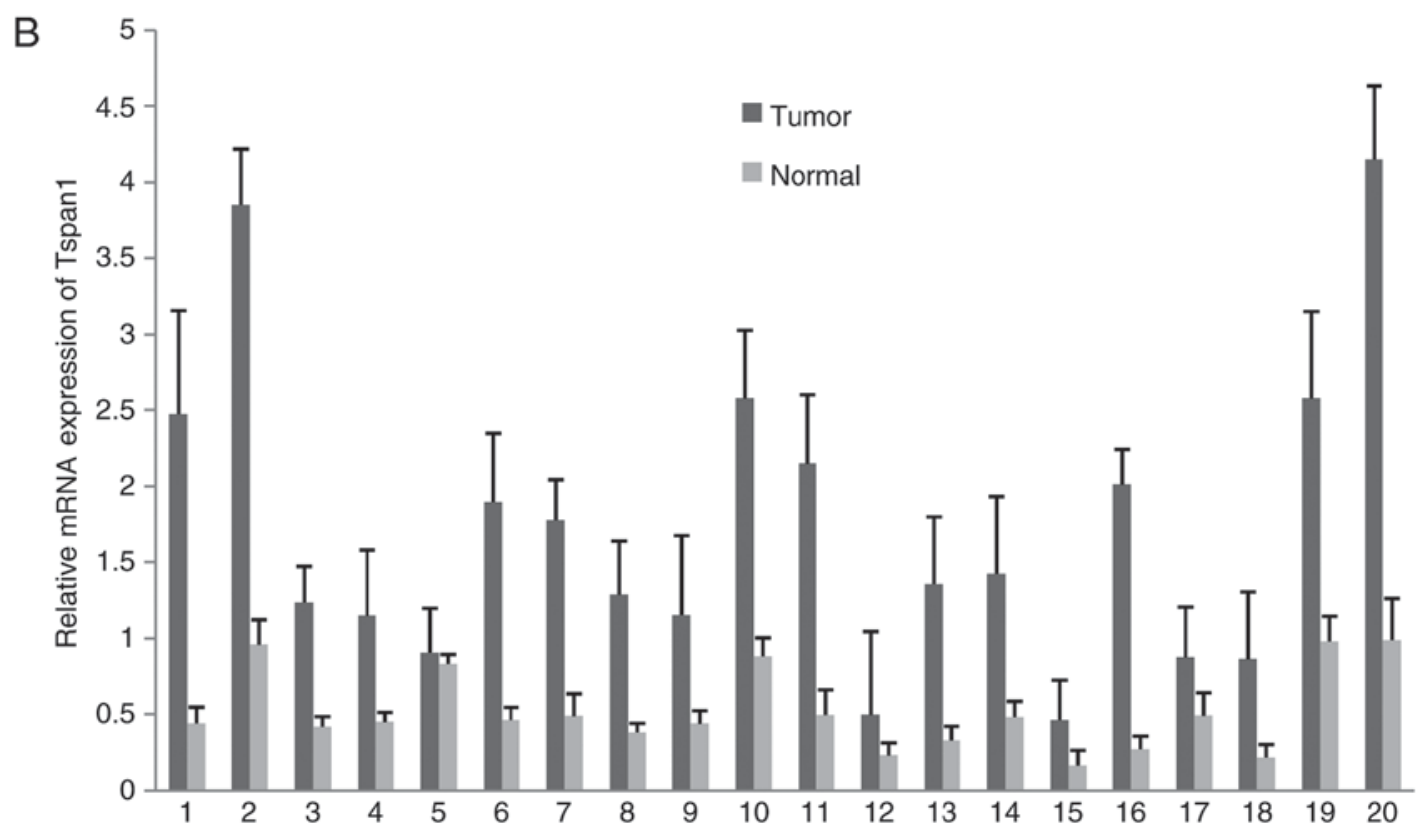

Figure 2. Statistical analysis results of Tspan1 expression in human pancreatic tumor tissues and adjacent normal pancreas tissues. Immunostaining and reverse transcription-quantitative polymerase chain reaction analyses of Tspan1 expression in 20 pairs of resection specimens from patients with pancreatic cancer. (A) The expression of Tspan1 in the adjacent normal pancreatic tissues and pancreatic cancer tissues by immunohistochemistry. (B) Relative Tspan1 mRNA levels in the adjacent normal pancreatic tissues (Normal) and pancreatic cancer tissues (Tumor). Intensity of staining was scored according to the following criteria: \pm (negative),+ (weak), ++ (intermediate) and +++ (strong). Data are expressed as the mean \pm standard deviation. TSPAN1, Tetraspanin 1 .

A
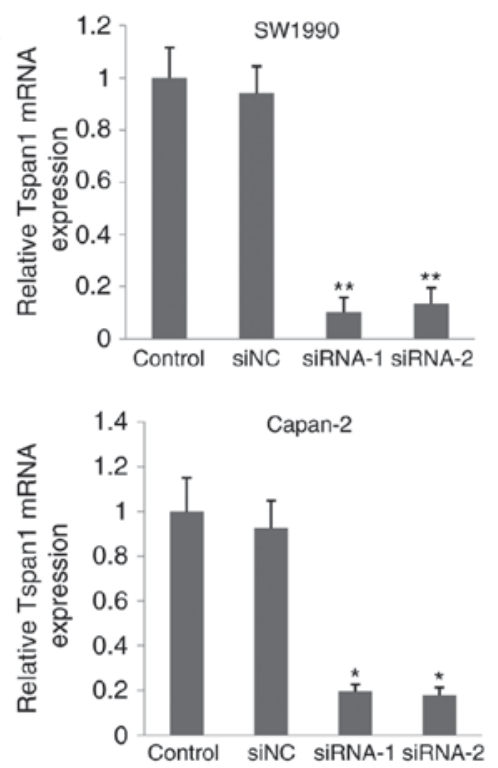

B SW1990 Control siNC SiRNA-1 SiRNA-2 TSPAN1 $\beta$-actin
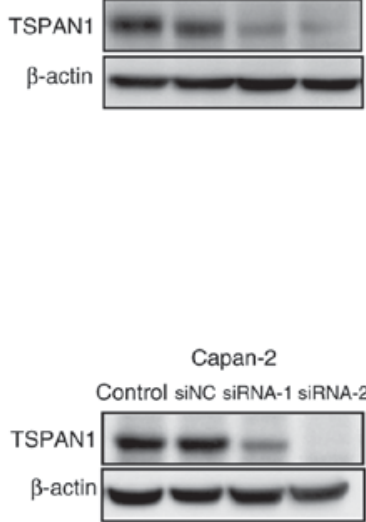
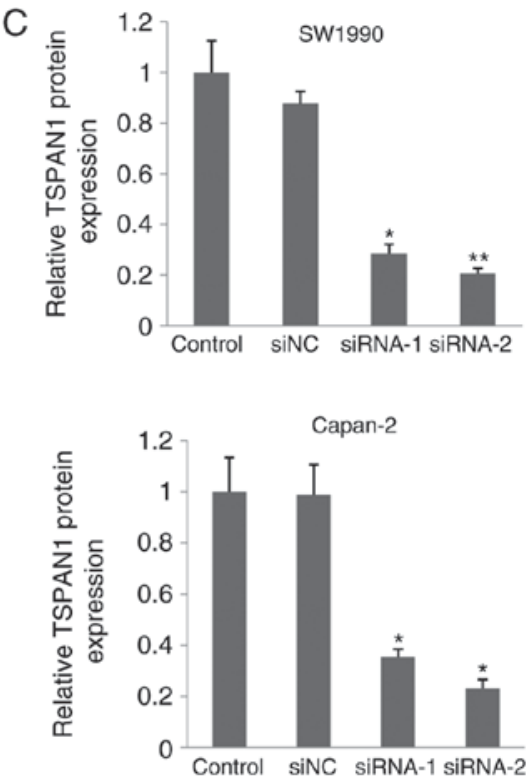

Figure 3. siTspan1 was transfected into SW1990 and Capan-2 cell lines successfully as confirmed by reverse transcription-quantitative polymerase chain reaction and western blot analysis. siRNAs targeted to Tspan1 resulted in decreased (A) mRNA and (B) protein expression levels in (C) SW1990 and Capan-2 cell lines when compared with the controls cells. Data are expressed as the mean \pm standard deviation. ${ }^{*} \mathrm{P}<0.05$ and ${ }^{* *} \mathrm{P}<0.01$ vs. control cells $(\mathrm{n}=3)$. si, small interfering; NC, negative control; TSPAN1, Tetraspanin 1. 
A
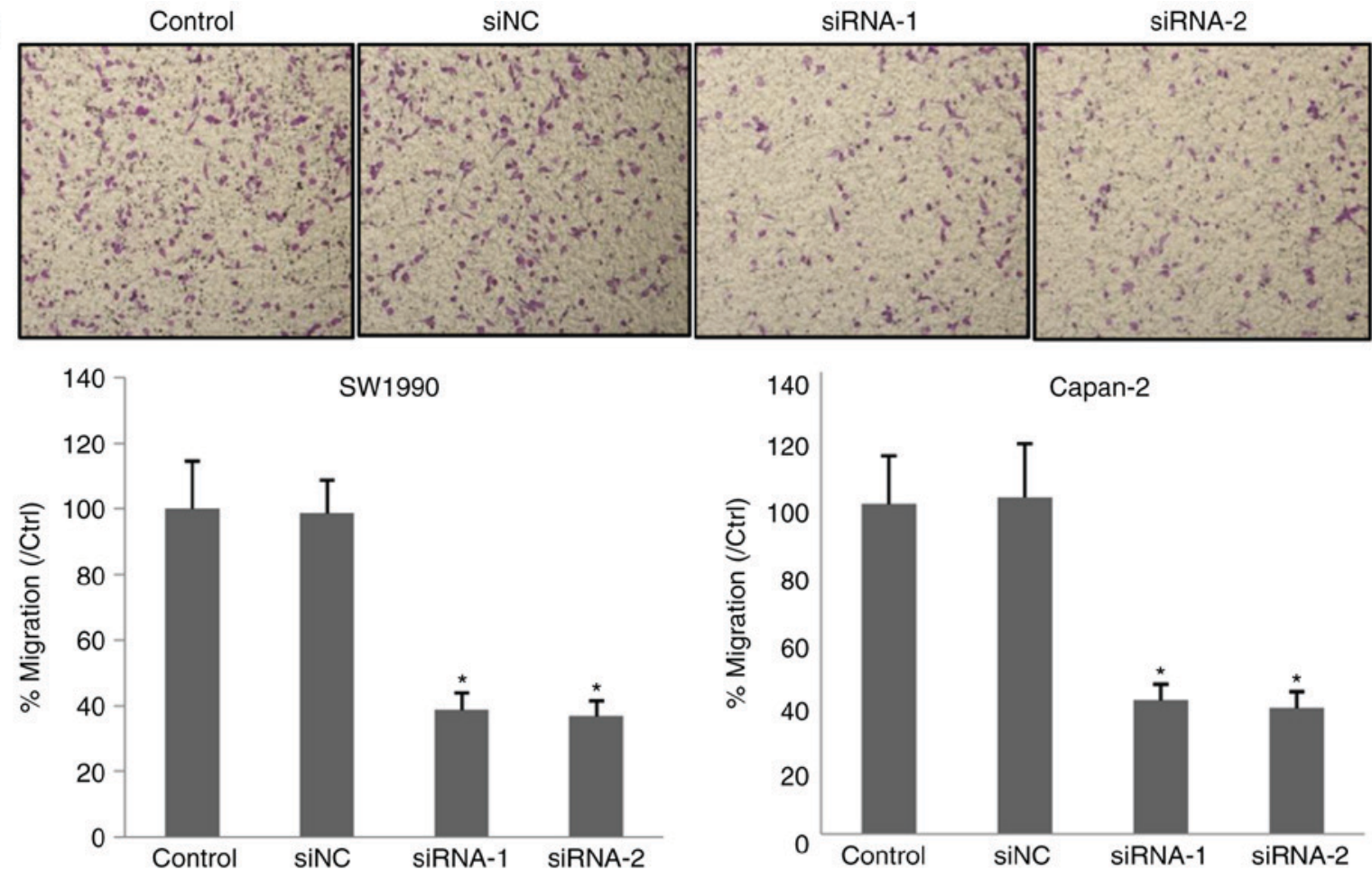

B
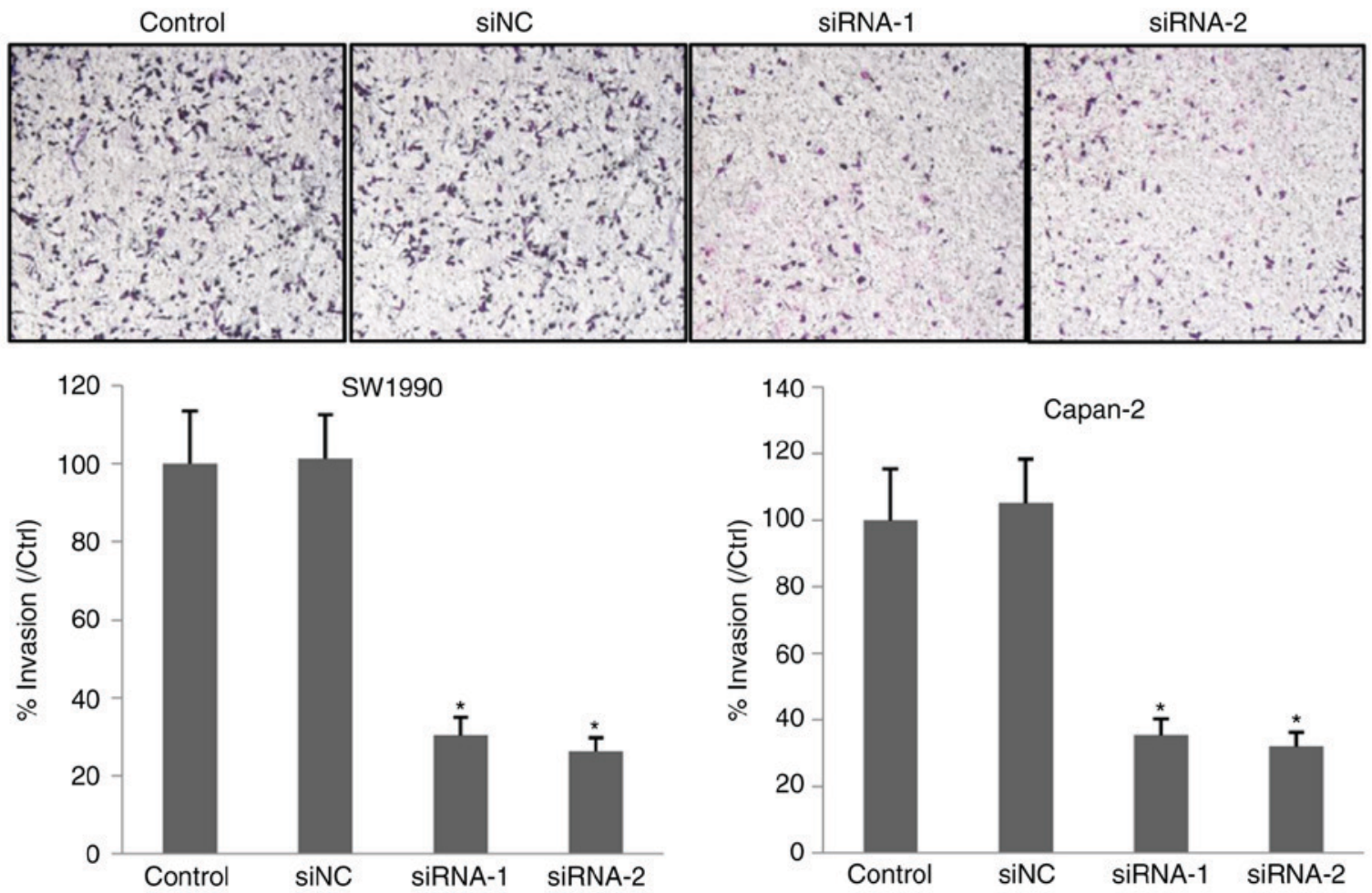

Figure 4. Transfection of siTspan1 attenuates the migration and invasion ability of SW1990 cells and Capan-2 cells in vitro. Representative images of (A) migrating and (B) invading SW1990 and Capan-2 cells; there were significantly less siRNA-transfected cells than control cells. Magnification, x100. Data are expressed as the mean \pm standard deviation. " $\mathrm{P}<0.05$ vs. control cells $(\mathrm{n}=3)$. si, small interfering; NC, negative control; TSPAN1, Tetraspanin 1.

demonstrated that Tspan1 mRNA expression was significantly knocked down in the Tspan1-siRNAs transfected cells compared with control cells that had not been transfected with siRNA (Fig. 3A). Western blotting results demonstrated that the protein levels of TSPAN1 were significantly lower in Tspan1-siRNAtransfected cells when compared with the control cells (Fig. 3B and C).
Knockdown of Tspan1 inhibits SW1990 and Capan-2 cell migration and invasion. FollowingTspan1-siRNAs transfection, the migration ability of Tspan1 knockdown cells, control cells and non-targeted siNC-transfected cells was determined and analyzed. The results demonstrated that in the Tspan1-siRNA1 and Tspan1-siRNA2 transfected groups the migrated cells were significantly decreased when compared 
A
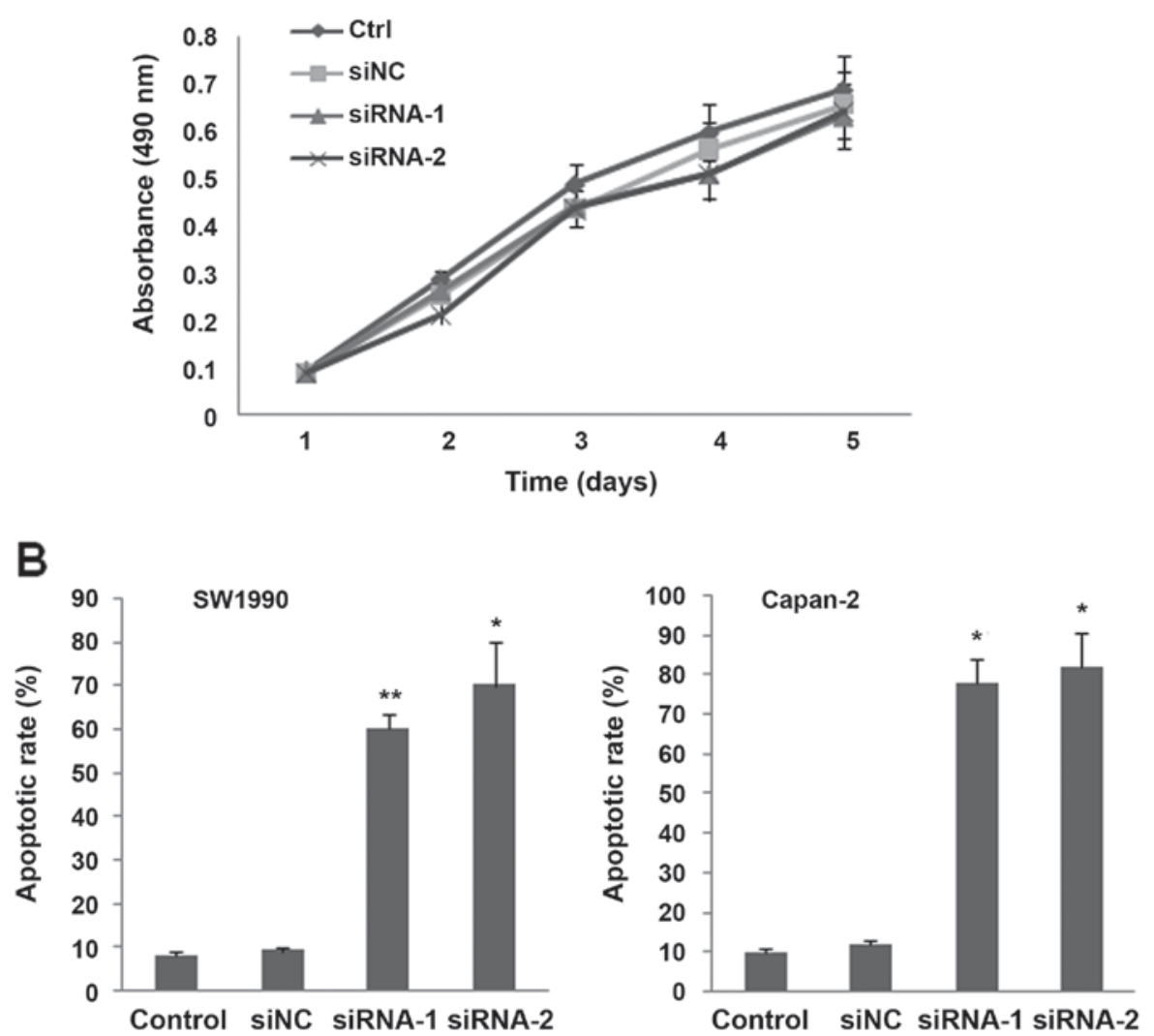

Figure 5. Transfection of siTspan1 induces cell apoptosis and cell survival in vitro. The influence of silencing Tspan1 on cell survival and cell apoptosis were detected by (A) MTT assay and (B) flow cytometry, respectively. Quantification demonstrated that the percentage of apoptotic cells in the Tspan1-siRNA transfected group was significantly higher when compared with the control groups. Data are expressed as the mean \pm standard deviation. ${ }^{*} \mathrm{P}<0.05$ and ${ }^{* * *} \mathrm{P}<0.01$ vs. control cells ( $\mathrm{n}=3$ ). si, small interfering; $\mathrm{NC}$, negative control; TSPAN1, Tetraspanin 1.
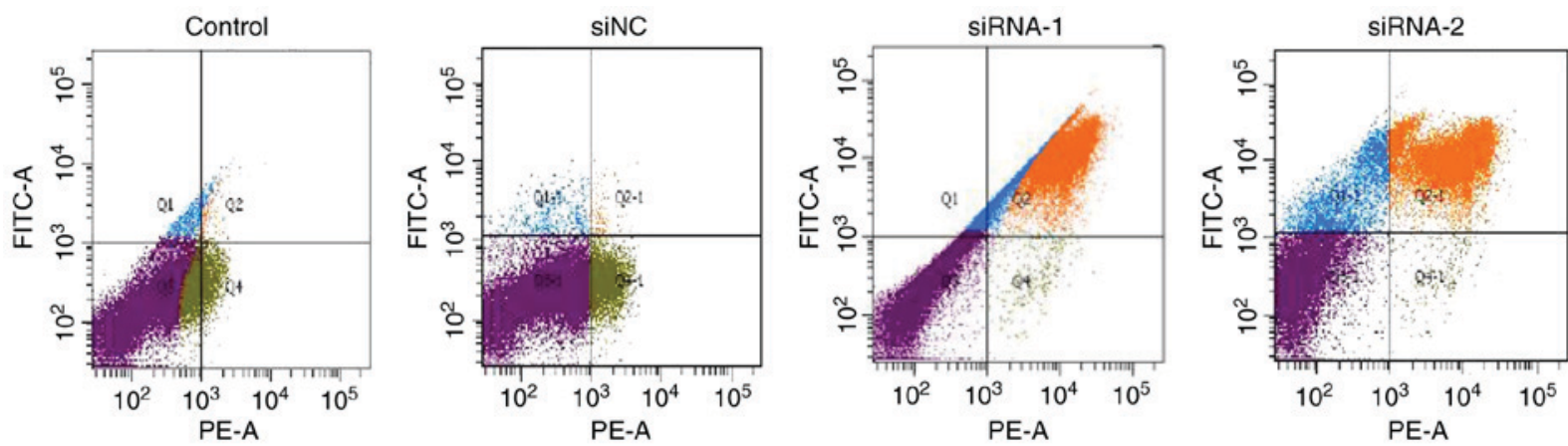

Figure 6. Original dot data of the Annexin V assay. SW1990 cell apoptosis was detected using Annexin V-FITC/PI staining assay by flow cytometry. Cells in the lower left quadrant represent surviving cells; the lower right quadrant represents early apoptosis; the upper right quadrant represents late apoptosis; and the upper left quadrant represents necrosis. The results demonstrated that when compared with the control group, the percentage of apoptosis increased in the Tetraspanin 1siRNA group. Data are expressed as the late apoptosis rate percentage. FITC, fluorescein isothiocyanate; PI, propidium iodide; si, small interfering; NC, negative control; PE-A, Phycoerythrin-A.

with that of the control (Fig. 4A). Subsequently, the invasion ability of Capan-2 and SW1990 cells following the knockdown of Tspan1 was determined using the Transwell invasion method. The results indicated that Tspan1-siRNA transfection significantly suppressed cell invasion (Fig. 4B). No significant differences in migration and invasion abilities between siNC cells and control cells were observed. Taken together, the results indicated that knockdown of Tspan1 inhibited cell migration and invasion.
Effects of Tspanl knockdown on cell survival and apoptosis. The effects of Tspan1 knockdown on cell survival and cell apoptosis were determined. The results of the MTT assay indicated that knockdown of Tspan1 did not exhibit a marked effect on cell survival (Fig. 5A). The results of flow cytometric analysis indicated that the percentage of apoptotic cells was increased in the Tspan1-siRNA transfection SW1990 groups, when compared the levels observed in the control groups $(60.16 \pm 3.33$ and $70.15 \pm 9.97 \%$ vs. $9.12 \pm 1.13$ and $8.37 \pm 1.24 \%$; Figs. 5 B and 6 ). 
The percentage of apoptotic cells was also increased in the Tspan1-siRNA transfection Capan-2 groups (data not shown).

\section{Discussion}

Despite the number of treatments available for patients with PCC including, surgery resection, radiation, chemotherapy and a combination of these modalities, PCC still has the worst prognosis among all of the other major tumors; following diagnosis, the 5 years survival rate is $<6 \%(1,4)$. Therefore, novel therapeutic strategies are urgently required.

It has been reported that tetraspanins, the transmembrane protein family, may be involved in the regulation of cell survival, growth, proliferation, differentiation, motility, and cancer cell migration and invasion $(9,11)$. In a number of human tumors, it has been demonstrated that the expression of tetraspanins was increased, which was associated with cancer progression (12). Tspan1 is a novel member of the tetraspanins family. Previous studies revealed that the expression of Tspan1 was increased or overexpressed in a variety of human carcinomas (14-18). The present study also demonstrated that the expression of TSPAN1 in human PCC was significantly higher than that observed in the adjacent normal pancreatic tissues. Therefore, these results may indicate that TSPAN1 expression could be used as a specific marker for human PCC in future strategies for diagnosis and treatment; however, further investigation is required to explore this hypothesis.

Previous studies have revealed that Tspan1 is involved in processes associated with tumor progression, including cell survival, proliferation, migration and invasion (14-18). Chen et al $(22,23)$ indicated that the knockdown of Tspan1 suppressed human colon cancer and skin carcinoma cell survival, proliferation, migration and invasion. Hölters et al (31) revealed that TSPAN1 promoted human cervical tumor cell migration and invasion. These studies implied that Tspan1 may serve an important role in cancer progression, while the detailed function of Tspan1 in human pancreatic tumor cells is still unclear. In the present study, the role of Tspan1 was explored in human PCC cells. The results indicated that the expression of Tspan1 was increased in human PCC tissues and the human PDAC cell lines Capan-2 and SW1990. Tspan1 expression was silenced following transfection with Tspan1 siRNA1 and siRNA2 into SW1990 and Capan-2 cells. The present findings indicated that silencing Tspan1 expression suppressed human pancreatic tumor cell migration and invasion, and induced the cell apoptosis of SW1990 and Capan-2 cells, which was consistent with previous findings in different tumor cells $(20,22,32)$. These findings indicated that Tspan1 may act as an oncogene. However, the MTT assay results indicated that knockdown of Tspan1 had no effect on cell viability and proliferation. It is possible that the remaining viable cells exhibited exceptionally high metabolic activity, therefore converting a comparable amount of MTT assay substrate as non-knockdown cells. In addition, it is also possible that the number of remaining live cells was too large, and the MTT was oversaturated. In future studies, the group will repeat these experiments and explore the underlying mechanisms.

Previous studies have demonstrated that Tspan1 and integrin $\alpha 6$ expression act as potential biomarkers in gastric, colonic, hepatic and esophageal carcinomas $(33,34)$; however, conflicting findings regarding their prognostic role have been reported in PDAC (35). The results of the present study demonstrated that Tspan1 was mainly expressed in the cytoplasm of human PCC cells. When Tspan 1 is located on the cell membrane it may interact with extracellular signals and carry out functions in the cytoplasm, which is similar to other Tetraspanins including CD63, CD82 and CD9 $(29,33,36)$. Thus future studies, including microarray analysis to investigate cancer associated genes regulated by Tspan1, and the underlying mechanism, are required.

In conclusion, the results of the present study indicated that Tspan1 expression was increased in human pancreatic tumor tissues and cell lines. The function of Tspan1 in human PCC progression was also investigated using Tspan1 siRNA. The results indicated that following the transfection of Capan-2 and SW1990 cells with Tspan1 siRNAs, cell migration and invasion were suppressed, and cell apoptosis was induced. These findings highlighted the important functional role of Tspan1 in human PCC progression. The results of the present study indicated that Tspan1 may be effective as a biomarker for PCC; however, further investigation is required to explore this hypothesis. siRNA targeting of Tspan1 may be a potential therapeutic strategy for the treatment of Tspan1 expressing pancreatic and other cancers, though further studies are required in the future.

\section{Acknowledgements}

Not applicable.

\section{Funding}

The present study was supported by the Science plan project of Shenyang Municipal Science and Technology Bureau (grant no. 18-014-4-75), the Liaoning Doctoral Scientific Research Initial Foundation (grant no. 201601417) and the National Natural Science Foundation (grant no. 81672427).

\section{Availability of data and materials}

The datasets used and/or analyzed during the present study are available from the corresponding author on reasonable request.

\section{Authors' contributions}

FL, JZho, MD and GS conceived and designed the present study. JT, RZ, GS, LX and WShe performed the experiments. HP, XZ, XY, WSha, JZha and XL analyzed the data. GS, FL, LX and XL wrote the manuscript. All authors read and approved the final manuscript.

\section{Ethics approval and consent to participate}

The present study was approved by the Ethical Committee of China Medical University (Shenyang, Liaoning, China). Written informed consent was obtained from each patient recruited.

\section{Patient consent for publication}

Written informed consent was obtained from each patient. 


\section{Competing interests}

The authors declare that they have no competing interests.

\section{References}

1. Vincent A, Herman J, Schuick R, Hruban RH and Goggins M: Pancreatic cancer. Lancet 378: 607-620, 2011.

2. Siegel R, Ma J, Zou Z and Jemal A: Cancer statistics, 2014. CA Cancer J Clin 64: 9-29, 2014

3. Bosetti C, Bertuccio P, Negri E, La Vecchia C, Zeegers MP and Boffetta P: Pancreatic cancer: Overview of descriptive epidemiology. Mol Carcinog 51: 3-13, 2012.

4. Loos M, Kleeff J, Friess H and Büchler MW: Surgical treatment of pancreatic cancer. Ann NY Acad Sci 1138: 169-180, 2008

5. Cascinu S, Berardi R, Labianca R, Siena S, Falcone A, Aitini E, Barni S, Di Costanzo F, Dapretto E, Tonini G, et al: Cetuximab plus gemcitabine and cisplatin compared with gemcitabine and cisplatin alone in patients with advanced pancreatic cancer: A randomised, multicentre, phase II trial. Lancet Oncol 9: 39-44, 2008.

6. Nakai Y, Isayama H, Sasaki T, Sasahira N, Tsujino T, Toda N, Kogure $\mathrm{H}$, Matsubara S, Ito $\mathrm{Y}$, Togawa $\mathrm{O}$, et al: A multicentrer and omised phase II trial of gemcitabine alone vs gemcitabine and S-1 combination therapy in advanced pancreatic cancer: GEMSAP study. Br J Cancer 106: 1934-1939, 2012.

7. Tuinmann G, Hegewisch-Becker S, Zschaber R, Kehr A, Schulz J and Hossfeld DK: Gemcitabine and mitomycin $C$ in advanced pancreatic cancer: A single-institution experience. Anticancer Drugs 15: 575-579, 2004.

8. Hall WA, Colbert LE, Nickleach D, Switchenko J, Liu Y, Gillespie T, Lipscomb J, Hardy C, Kooby DA, Prabhu RS, et al: The influence of radiation therapy dose escalation on overall survival in unresectable pancreatic adenocarcinoma. J Gastrointest Oncol 5: 77-85, 2014.

9. Veenbergen S and Van Spriel AB: Tetraspanins in the immune response against cancer. Immunol Lett 138: 129-136, 2011.

10. Berditchevski F, Odintsova E, Sawada S and Gilber E: Expression of the palmitoylation-deficient CD151 weakens the association of alpha 3 beta 1 integrin with the tetraspanin-enriched microdomains and affects integrin dependent signaling. J Biol Chem 277: 36991-37000, 2002.

11. Levy S and Shoham T: The tetraspanin web modulates immunesignalling complexes. Nat Rev Immunol 5: 136-148, 2005.

12. Pedro AL: Functional implications of tetraspanin proteins in cancer biology. Cancer Sci 98: 1666-1677, 2007.

13. Serru V, Dessen P, Boucheix C and Rubinstein E: Sequence and expression of seven new tetraspans. Biochim Biophys Acta 1478 $159-163,2000$

14. Scholz CJ, Kurzeder C, Koretz K, Windisch J, Kreienberg R, Sauer G and Deissler H: Tspan-1 is a tetraspanin preferentially expressed by mucinous and endometrioid subtypes of human ovarian carcinomas. Cancer Lett 275: 198-203, 2009.

15. Chen L, Zhu YY, Zhang XJ, Wang GL, Li XY, He S, Zhang JB and Zhu JW: TSPAN1 protein expression: A significant prognostic indicator for patients with colorectal adenocarcinoma. World J Gastroenterol 15: 2270-2276, 2009.

16. Chen L, Yuan D, Wang GL, Wang Y, Wu YY and Zhu J: Clinicopathological significance of expression of Tspan-1, Jab1 and p27 in human hepatocellular carcinoma. J Korean Med Sci 25: 1438-1442, 2010.

17. Chen L, Li X, Wang GL, Wang Y, Zhu YY and Zhu J: Clinicopathological significance of overexpression of TSPAN1, Ki67 and CD34 in gastric carcinoma. Tumori 94: 531-538, 2008.

18. Wollscheid V, Kühne-Heid R, Stein I, Jansen L, Köllner S, Schneider A and Dürst M: Identification of a new proliferation associated protein NET-1/C4.8 characteristic for a subset of high-grade cervical intraepithelial neoplasia and cervical carcinomas. Int J Cancer 99: 771-775, 2002.
19. Xu J, Stolk JA, Zhang X, Silva SJ, Houghton RL, Matsumura M, Vedvick TS, Leslie KB, Badaro R and Reed SG: Identification of differentially expressed genes in human prostate cancer using subtraction and microarray. Cancer Res 60: 1677-1682, 2000.

20. Liu L, He B, Liu WM, Zhou D, Cox JV and Zhang XA: Tetraspanin CD151 promotes cell migration by regulating integrin trafficking. J Biol Chem 282: 31631-31642, 2007.

21. Yamamoto $\mathrm{Y}$, Grubisic $\mathrm{K}$ and Oelgeschläger $\mathrm{M}$ : Xenopus tetraspanin-1 regulates gastrulation movements and neural differentiation in the early xenopus embryo. Differentiation 75 : 235-245, 2007

22. Chen L, Yuan D, Zhao R, Li H and Zhu J: Suppression of TSPAN1 by RNA interference inhibits proliferation and invasion of colon cancer cells in vitro. Tumori 96: 744-750, 2010.

23. Chen L, Zhu Y, Li H, Wang GL, Wu YY, Lu YX, Qin J, Tuo J, Wang JL and Zhu J: Knockdown of TSPAN1 by RNA silencing and antisense technique inhibits proliferation and infiltration of human skin squamous carcinoma cells. Tumori 96: 289-295, 2010.

24. Carlson RW, Hudis CA and Pritchard KI: Adjuvant endocrine therapy in hormone receptor-positive postmenopausal breast cancer: Evolution of NCCN, ASCO, and St Gallen recommendations. J Natl Compr Canc Netw 4: 971-979, 2006.

25. Desouki MM, Liao S, Huang H, Conroy J, Nowak NJ, Shepherd L, Gaile DP and Geradts J: Identification of metastasisassociated breast cancer genes using a high-resolution whole genome profiling approach. J Cancer Res Clin Oncol 137: 795-809, 2011.

26. Liu H, Ma Q and Li J: High glucose promotes cell proliferation and enhances GDNF and RET expression in pancreatic cancer cells. Mol Cell Biochem 347: 95-101, 2011.

27. Livak KJ and Schmittgen TD: Analysis of relative gene expression data usingreal-time quantitative PCR and the 2(-Delta Delta C(T)) method. Methods 25: 402-408, 2001

28. Albrethsen J, Bøgebo R, Gammeltoft S, Olsen J, Winther B and Raskov H: Upregulated expression of human neutrophil peptides 1,2 and 3 (HNP1-3) in colon cancer serum and tumours: A biomarker study. BMC Cancer 5: 8, 2005.

29. Fei Y, Wang J, Liu W, Zuo H, Qin J, Wang D, Zeng H and Liu Z: CD151 promotes cancer cell metastasis via integrins $\alpha 3 \beta 1$ and a6 $\beta 1$ in vitro. Mol Med Rep 6: 1226-1230, 2012.

30. Takahashi M, Sugiura T, Abe M, Ishii K and Shirasuna K: Regulation of c-Met signaling by the tetraspanin KAI-1/CD82 affects cancer cell migration. Int J Cancer 121: 1919-1929, 2007.

31. Hölters S, Anacker J, Jansen L, Beer-Grondke K, Dürst M and Rubio I: Tetraspanin 1 promotes invasiveness of cervical cancer cells. Int J Oncol 43: 503-512, 2013.

32. Lu Z, Luo T, Nie M, Pang T, Zhang X, Shen X, Ma L1, Bi J, Wei G, Fang G and Xue X: TSPAN1 functions as an oncogene in gastric cancer and is downregulated by miR-573. FEBS Lett 589: 1988-1994, 2015.

33. Wang H, Rana S, Giese N, Büchler MW and Zöller M: Tspan8, $\mathrm{CD} 44 \mathrm{v} 6$ and alpha6beta4 are biomarkers of migrating pancreatic cancer-initiating cells. Int J Cancer 133: 416-426, 2013.

34. Schmelz M, Cress AE, Scott KM, Bürger F, Cui H, Sallam K, McDaniel KM, Dalkin BL and Nagle RB: Different phenotypes in human prostate cancer: Alpha6 or alpha3 integrin in cell-extracellular adhesion sites. Neoplasia 4: 243-254, 2002.

35. Herlevsen M, Schmidt DS, Miyazaki K and Zöller M: The association of the tetraspanin D6.1A with the alpha6beta4 integrin supports cell motility and liver metastasis formation. J Cell Sci 116: 4373-4390, 2003.

36. Chen Z, Mustafa T, Trojanowicz B, Brauckhoff M, Gimm O, Schmutzler C, Köhrle J, Holzhausen HJ, Kehlen A, Klonisch T, et al: CD82, and CD63 in thyroid cancer. Int J Mol Med 14: 517-527, 2004.

This work is licensed under a Creative Commons Attribution-NonCommercial-NoDerivatives 4.0 International (CC BY-NC-ND 4.0) License. 